\title{
Radiation Pressure Cooling of a Micromechanical Oscillator Using Dynamical Backaction
}

\author{
A. Schliesser, ${ }^{1}$ P. Del'Haye ${ }^{1}{ }^{\text {N. Nooshi, }}{ }^{1}$ K. J. Vahala, ${ }^{2, *}$ and T. J. Kippenberg ${ }^{1, \dagger}$ \\ ${ }^{1}$ Max Planck Institut für Quantenoptik, 85748 Garching, Germany \\ ${ }^{2}$ Department of Applied Physics, California Institute of Technology, Pasadena, California 91125 USA
}

(Received 24 October 2006; published 14 December 2006)

Cooling of a $58 \mathrm{MHz}$ micromechanical resonator from room temperature to $11 \mathrm{~K}$ is demonstrated using cavity enhanced radiation pressure. Detuned pumping of an optical resonance allows enhancement of the blueshifted motional sideband (caused by the oscillator's Brownian motion) with respect to the redshifted sideband leading to cooling of the mechanical oscillator mode. The reported cooling mechanism is a manifestation of the effect of radiation pressure induced dynamical backaction. These results constitute an important step towards achieving ground state cooling of a mechanical oscillator.

Cooling of micromechanical resonators such as cantilevers is an important prerequisite for studies ranging from highly sensitive measurements of forces [1] and displacement [2] to observing quantum mechanical phenomena of macroscopic objects [3] and gravitational wave detection. Early work recognized the possibility to cool a mechanical resonator mode by radiation pressure, via use of an active feedback scheme [4,5], in close analogy to stochastic cooling of charged particles. However, techniques which cool a mechanical oscillator intrinsically via radiation pressure, as routinely achieved in atomic laser cooling while proposed theoretically [6-8] - have never been demonstrated. Here we report such a technique based on radiation pressure [8] and apply it to cool a $58-\mathrm{MHz}$ micromechanical oscillator in the form of a toroid cavity from room temperature to $11 \mathrm{~K}$.

The present work falls within the setting of high-finesse optomechanical systems, which couple a mechanical oscillator (such as a cantilever [9-11], an internal mode of a mirror [4] or a microcavity [12]) to an optical cavity field by means of a high-finesse optical resonator as shown schematically in Fig. 1(b). The motion of the mirror renders the cavity transmission position dependent. Indeed, the use of a Fabry-Pérot interferometer is among the most sensitive methods for displacement measurements [6] and the underlying principle of the Laser Interferometer Gravitational Wave Observatory (LIGO). This monitoring, however, can become unstable owing to radiation pressure, which can lead to regenerative oscillations of the mechanical eigenmodes as first predicted by Braginsky [13]. This phenomenon is a manifestation of the effect of dynamical backaction [6,14]. In fact, while effects of radiation pressure related phenomena (such as bistability [15]) have been observed for more than two decades - and have been subject of various theoretical studies $[3,5,16]$ - the dynamical backaction caused by radiation pressure has only recently been observed in toroid microcavities on a chip $[7,12,17]$. These toroidal microresonators [18,19] (cf. Fig. 1) possess ultrahigh- $Q$ whispering gallery type optical modes while simultaneously exhibiting microme- chanical resonances in the radio-frequency domain. Because of the curved nature of the dielectric cavity, the circulating photons exert a radial force on the cavity sidewalls, thereby coupling the optical and mechanical degree of freedom, analogous to a Fabry-Pérot cavity with a movable mirror [Fig. 1(b)]. Owing to the resonator's optical photon lifetime being similar to the mechanical oscillation period, these devices operate in a regime where cavity retardation effects cannot be neglected, which has enabled the study of radiation pressure induced oscillations $[7,12,17]$ and related effects $[6,20]$ in an experimental setting. While dynamical backaction can lead to mechanical oscillations [12], it can also, as shown here, lead to cooling of the mechanical mode as predicted in earlier work $[7,8]$.

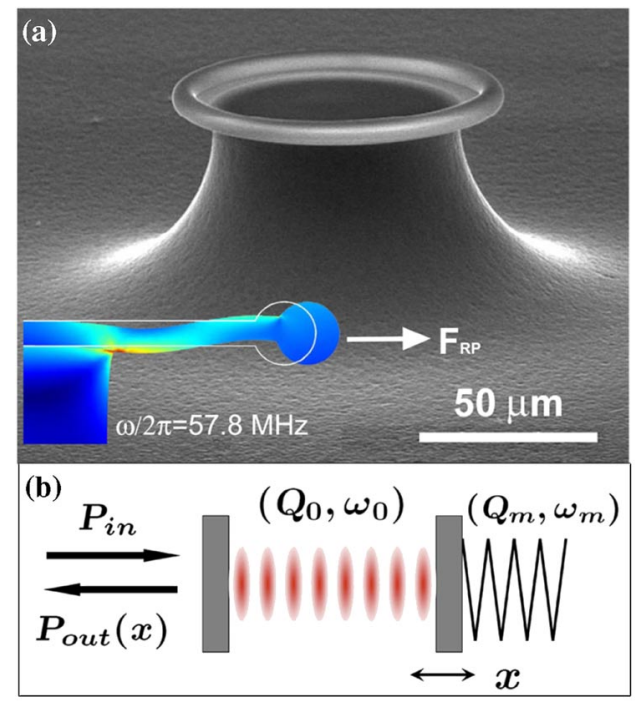

FIG. 1 (color online). Main panel: A scanning electron micrograph of a toroid microcavity on a chip. Inset: Finite-element simulation showing the stress (color coded) and strain of the 57.8-MHz breathing mode of the device. The strain is exaggerated for clarity. Note that radiation pressure exerts a radial force owing to total internal reflection of the confined light. (b) A Fabry-Pérot equivalent diagram of the experiments. 
The mechanical mode under consideration in this study is a radial breathing mode with resonance frequency $\left(f_{m}=\right.$ $\left.\omega_{m} / 2 \pi\right)$ of $57.8 \mathrm{MHz}$. Since the mechanical mode is in thermal equilibrium with the environment $(T=300 \mathrm{~K})$, it follows from the classical equipartition theorem that the mechanical oscillator undergoes Brownian motion with a root mean square displacement of $\left\langle x^{2}\right\rangle^{1 / 2}=\sqrt{\frac{k_{B} T}{m \omega_{m}^{2}}}$, where $k_{B}$ is the Boltzmann constant. For the present microresonator, whose effective mass is $m_{\mathrm{eff}}=1.5 \times 10^{-11} \mathrm{~kg}$ (as determined independently by finite-element simulation) this amplitude corresponds to $\sim 5 \times 10^{-14} \mathrm{~m}$. Despite the small amplitude the thermally excited oscillations are readily observable in the transmitted light, when a cavity mode is excited using a tapered optical fiber. Operating the cavity detuned, the amplitude fluctuations of the mechanical resonator cause a change in the cavity transmission that enables extraction of the mechanical resonator characteristics (resonance frequency $\omega_{m} / 2 \pi$, linewidth $\gamma / 2 \pi=$ $\omega_{m} / 2 \pi Q_{m}$, and displacement spectral density $\left.\left\langle x_{\tilde{\omega}}^{2}\right\rangle\right)$. Figure 2 shows displacement monitoring of the microresonator obtained for varying power levels at a constant red detuning of the laser frequency $\omega / 2 \pi$, with respect to the cavity resonance, $\omega_{0} / 2 \pi$. The normalized detuning $\Delta \omega \tau=\left(\omega-\omega_{0}\right) \tau$, where $\tau$ is the photon lifetime, is ca. -0.5 for these measurements. From fits to the noise spectra at low pump power $(10 \mu \mathrm{W})$, the resonance frequency of $57.8 \mathrm{MHz}$ and an intrinsic mechanical quality factor $\left(Q_{m}\right)$ of 2890 were extracted. The optical linewidth at critical coupling was $\sim 50 \mathrm{MHz}$, equivalent to an optical
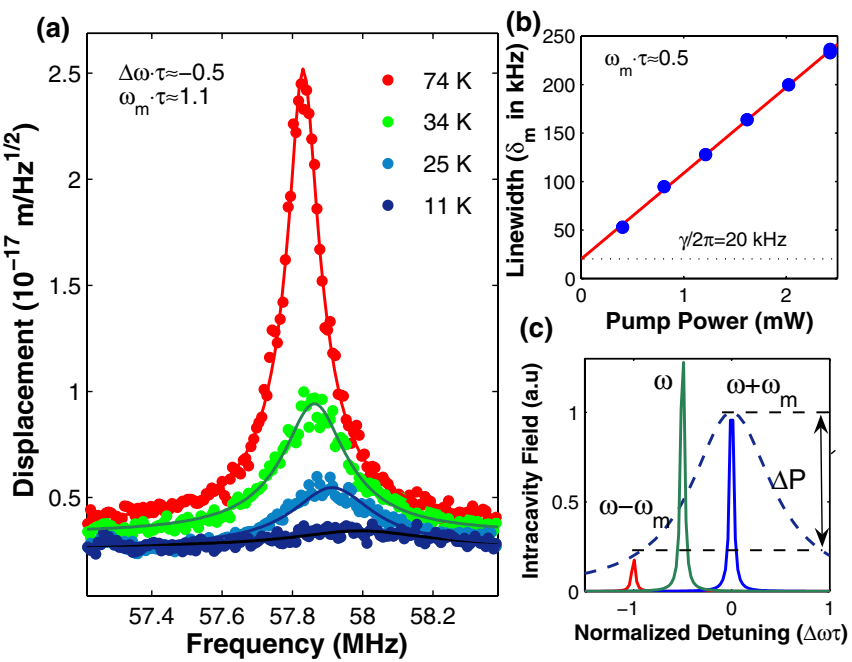

FIG. 2 (color online). Main figure shows the normalized, measured noise spectra around the mechanical resonance frequency for $\Delta \omega \tau \approx-0.5$ and varying power $(0.25,0.75,1.25$, and $1.75 \mathrm{~mW}$ ). The effective temperatures were inferred using mechanical damping, with the lowest attained temperature being $11 \mathrm{~K}$. (b) Increase in the linewidth (damping) of the $57.8-\mathrm{MHz}$ mode as a function of launched power, exhibiting the expected linear behavior [cf. Eqs. (1)]. (c) Physical origin of the observed cooling mechanism due to the asymmetry in the motional sidebands. quality factor of $Q=\omega_{0} \tau=6.2 \times 10^{6}$. Strikingly, when increasing the power of the red-detuned laser, a strong decrease in thermal displacement was observed (Fig. 2, main panel). A concomitant strong increase in the mechanical linewidth (damping) was also observed [cf. Fig. 2(b)], which in the present case can be used to infer an upper limit on the effective oscillator temperature [4]. Indeed, we confirmed that the area underneath the Lorentzian noise spectrum reduces by at least a factor $\times \frac{\gamma}{\gamma_{\text {eff }}}\left(\right.$ where $\gamma_{\text {eff }}$ is the effective linewidth [4]), which is a further measure of the effective oscillator temperature (since $T_{\text {eff }} k_{B}=\int m_{\mathrm{eff}}\left\langle x_{\tilde{\omega}}^{2}\right\rangle \tilde{\omega}^{2} d \tilde{\omega}$ ). For the highest pump power $(\sim 2 \mathrm{~mW}$ at $970 \mathrm{~nm})$, the effective temperature was reduced from 300 to $11 \mathrm{~K}$.

To substantiate our claim that indeed radiation pressure is responsible for the observed cooling, a theoretical coupled mode model based on radiation pressure is used [7] and compared to our findings. The oscillating cavity (with frequency $\omega_{m}$ ) produces both Doppler up-shifted $\left(\omega_{\mathrm{AS}}=\omega+\omega_{m}\right)$ anti-Stokes (AS) photons and downshifted $\left(\omega_{\mathrm{S}}=\omega-\omega_{m}\right)$ Stokes (S) photons (equivalent to the motional sidebands of trapped ions). If the cavity is pumped red detuned (i.e., $\omega<\omega_{0}$ ), the cavity resonance will suppress scattering into the red sideband, while enhance scattering into the blue sideband. This situation is depicted schematically in Fig. 2(c). This asymmetry in the sidebands leads to a net transfer of power from the mechanical oscillator to the light field and causes cooling of the mechanical resonator mode. The cooling power can be derived by noting that the generated Stokes and anti-Stokes sidebands produce a time varying radiation pressure force, whose quadrature component upon red detuning can extract power $\left(\tilde{P}=\left\langle\frac{d x}{d t} F_{\mathrm{RP}}\right\rangle\right)$ from the mechanical oscillator mode. The corresponding cooling rate $\left(\Gamma=-\frac{\tilde{P}}{\langle E\rangle}\right)$ is given by

$$
\Gamma=\frac{-F^{2} 8 n^{2} \omega_{0}}{m c^{2} \omega_{m}} C\left(\frac{1}{4 \tau^{2} \Delta \omega_{s}^{2}+1}-\frac{1}{4 \tau^{2} \Delta \omega_{\mathrm{as}}^{2}+1}\right) P_{\mathrm{in}} .
$$

Here $P_{\text {in }}$ denotes the launched power into the fiber, $F$ denotes finesse, and $1 / \tau_{\mathrm{ex}}$ is the rate of coupling into the cavity from the fiber and $\Delta \omega$ the laser detuning. Furthermore, the coupling parameter $C$ is introduced, $C \equiv$ $\frac{\tau / \tau_{\mathrm{ex}}}{4 \tau^{2} \Delta \omega^{2}+1} \in[0 . .1]$ and $m_{\mathrm{eff}}$ is the effective mass of the mechanical mode under consideration. The detuning of the Stokes and anti-Stokes photons are given by $\Delta \omega_{\mathrm{S}}=$ $\Delta \omega-\omega_{m}$ and $\Delta \omega_{\mathrm{AS}}=\Delta \omega+\omega_{m}$. We emphasize that efficient cooling is characterized by $\omega_{m} \tau \approx 1$. It is interesting to note that the physical process which gives rise to cooling is analogous to cooling of atoms or molecules via coherent scattering $[21,22]$. The final temperature of the mechanical oscillator in the presence of cooling is given by the balance of heating by the reservoir $\left(\langle P\rangle=\omega_{m} \frac{k_{B} T_{R}}{Q_{m}}\right.$, as described by the fluctuation dissipation theorem) and the radiation pressure induced cooling rate [4]: 


$$
\frac{T_{\mathrm{eff}}}{T_{R}} \approx \frac{\gamma}{\gamma+\Gamma}
$$

The presence of cooling thus changes the observed linewidth of the mechanical resonances $\left(\gamma_{\mathrm{eff}}=\gamma+\Gamma\right)$ and causes a reduction in the Brownian amplitude. In addition, the in-phase component of radiation pressure gives rise to a change in mechanical resonance frequency $\left(\omega_{\text {eff }}=\omega_{m}+\right.$ $\left.\Delta \omega_{m}\right)$ :

$$
\Delta \omega_{m}=\frac{8 n^{2} F^{2} \omega_{0}}{m_{\mathrm{eff}} c^{2} \omega_{m}} \tau C\left(\frac{\Delta \omega_{s}}{4 \tau^{2} \Delta \omega_{s}^{2}+1}+\frac{\Delta \omega_{\mathrm{as}}}{4 \tau^{2} \Delta \omega_{\mathrm{as}}^{2}+1}\right) P_{\mathrm{in}} .
$$

To verify these additional theoretical predictions a series of experiments was performed. First, the dependence of the cooling rate $\Gamma$ on the detuning was verified [Eq. (1) and Fig. 3]. For negative detuning a clear increase in the mechanical linewidth is observed (i.e., cooling) and for positive detuning a decrease in linewidth. Furthermore, for blue detuning, regions exist in which the radiation pressure induced amplification rate exceeds the intrinsic mechanical dissipation rate $\gamma$, leading to the regime of parametric oscillation instability $[7,12,17]$. Using Eq. (1) and leaving only the effective mass a free parameter (which agreed well with the simulated value), accurate agreement with the experimentally observed change in mechanical linewidth (damping) was obtained (solid line Fig. 3). This represents the first experimental evidence that indeed radiation pressure is responsible for the observed cooling.

Next, the modifications to the rigidity of the oscillator were measured. Equation (3) predicts that for $1 / \tau>2 \omega_{m}$ a decrease (increase) of the mechanical frequency in the cooling (amplification) regime. However, if the cavity bandwidth satisfies, $1 / \tau<2 \omega_{m}$, an unexpected behavior is predicted; the oscillator frequency will increase when cooled for small detuning, while for the remaining detuning it is shifted to lower frequencies. These predictions are in excellent agreement with the reported experimental results as shown in Fig. 3. Keeping the same sample but using a different optical resonance with a line-width of $113 \mathrm{MHz}$ (57.8 MHz mechanical resonance), the transition to a pure negative and positive frequency shift in the cooling and amplification regimes was observed as shown in Fig. 3(b), confirming the validity of our theoretical model.

While prior work has already established that radiation pressure is the dominant mechanism that drives mechanical oscillations in toroid microcavities $[7,12,17]$, measurements were also carried out to assess the contribution of thermal effects $[11,23]$. Thermal effects can cause regenerative mechanical oscillation and even cooling [11]. In order to quantify the thermal contribution at $57.8 \mathrm{MHz}$, the response of the cavity to a modulated pump in the frequency range of 0-200 MHz was recorded (see Fig. 4). This was achieved by recording the shift in optical resonance due to a modulated laser using a pump-probe scheme

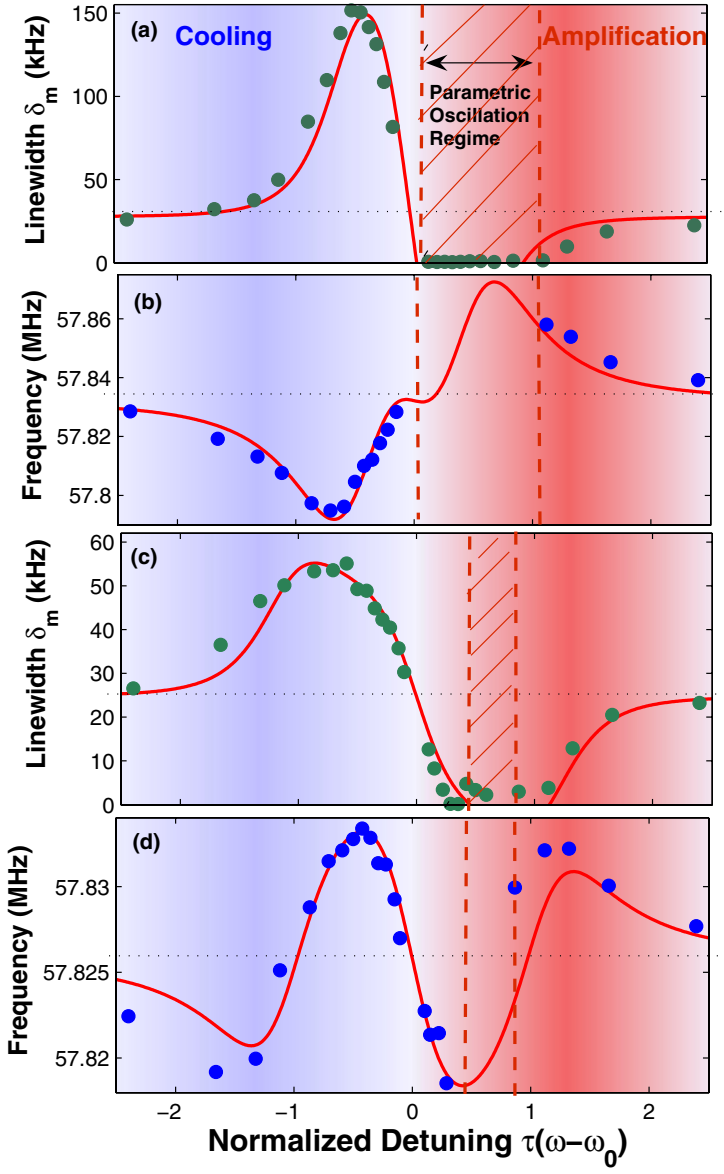

FIG. 3 (color online). Mechanical linewidth (a) and mechanical resonance frequency (b) of the $57.8-\mathrm{MHz}$ radial breathing mode as a function of (normalized) detuning when the laser is tuned over a $113 \mathrm{MHz}$ wide optical cavity resonance (corresponding to $\omega_{m} \tau=0.5$ ). The optical power was $P=1.0 \mathrm{~mW}$. The dashed region denotes occurrence of the parametric oscillation instability. For $\Delta \omega<0$ the $58-\mathrm{MHz}$ resonance is being cooled. Plots (c),(d) show the characteristics $\left(\omega_{m} / 2 \pi\right.$ and linewidth) of the same mechanical mode, but for scanning over a narrower (50 MHz linewidth) optical resonance (corresponding to $\omega_{m} \tau \approx 1.1$ ) with $P=0.13 \mathrm{~mW}$. Solid lines are theoretical predictions based on Eqs. (1)-(3). The only free parameter was the effective mass, which agreed well with the simulated value.

allowing us to differentiate different path-length-changing contributions due to the thermal nonlinearities, radiation pressure, and the Kerr effect based on their distinct frequency response. The poles in the response at 1.6 and $119 \mathrm{kHz}$ agree well with convective heat exchange to the $\mathrm{N}_{2}$ environment surrounding the resonator and thermal conduction to silica. For frequencies beyond $1 \mathrm{MHz}$, a plateau appears which is due to the (instantaneous) Kerr nonlinearity as observed in prior work [24]. At $58 \mathrm{MHz}$ the radiation pressure driven mechanical response is observable, which (inset Fig. 4) is a factor of $\times 260$ stronger than the Kerr nonlinearity in the present device. It is emphasized that this ratio is in quantitative agreement with the theoretically predicted Kerr-to-radiation pressure ratio. From 


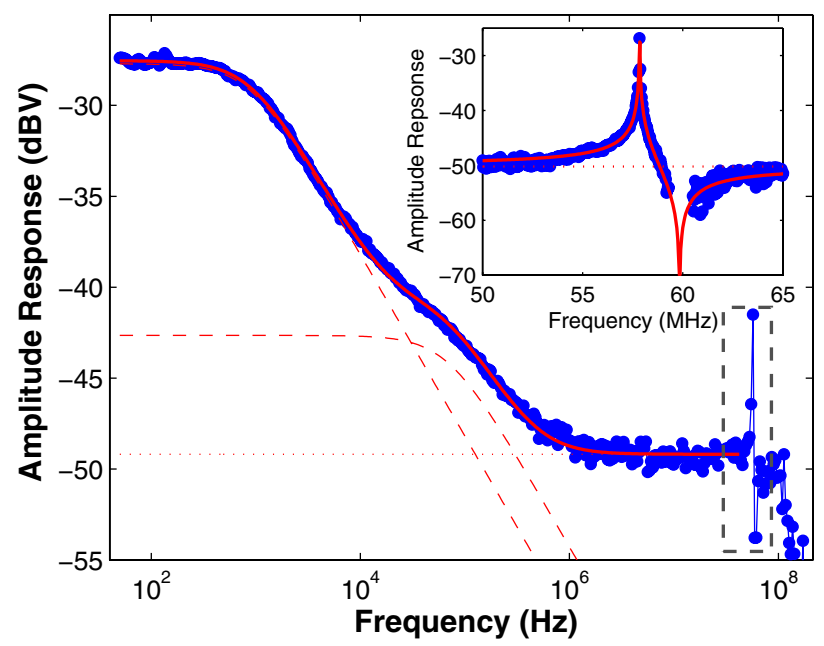

FIG. 4 (color online). Main panel: The frequency response from $0-200 \mathrm{MHz}$. The plateau occurring between $1-200 \mathrm{MHz}$ is due to the (instantaneous) Kerr nonlinearity of $\mathrm{SiO}_{2}$ (dotted line). The cutoff at $200 \mathrm{MHz}$ is due to both detector and cavity bandwidth. Inset: magnification of main panel in the vicinity of radiation pressure response which shows the interference of the Kerr nonlinearity and the radiation pressure-driven micromechanical resonator (which, on resonance, is $\pi / 2$ out of phase with the modulating pump and the instantaneous Kerr nonlinearity). From the fits (solid lines) it can be inferred that the radiation pressure response is ca. $\times 260$ larger than the Kerr response.

the well-identified frequency response of the aforementioned thermal effects we can furthermore conclude that their contribution to the interaction of the cavity field with the $57.8-\mathrm{MHz}$ radial breathing mode is at least 2 orders of magnitude too weak to explain the observed effects. Consequently, the response measurements give unambiguous proof that such thermal effects contribute less than 1 part in 100 to the observed cooling (or heating) rate.

In summary, we have reported radiation pressure induced cavity cooling of a radio-frequency $(57.8-\mathrm{MHz})$ micromechanical oscillator. Given the recent progress in high-finesse optomechanical systems it is reasonable to assume that this phenomenon will become observable in a wide variety of systems, and might provide a route to achieve ground-state cooling of a micromechanical oscillator.

This work was funded via a Max Planck Independent Junior Research Group grant, a EU Marie Curie Grant (No. CMIRG-CT-2005-031141) and the DFG (NIM Initiative). The authors gratefully acknowledge J. Kotthaus for clean room access and J. Alnis for technical assistance. T. W. Hänsch, K. Karrai, and W. Zwerger are thanked for stimulating discussions.

Note added. - After submission of this work, radiation pressure cooling of a micromechanical mirror by dynamical backaction [25,26] and active feedback [27] was reported.

*Electronic address: vahala@caltech.edu

†Electronic address: tjk@mpq.mpg.de

[1] D. Rugar, R. Budakian, H.J. Mamin, and B.W. Chui, Nature (London) 430, 329 (2004).

[2] A. N. Cleland and M. L. Roukes, Nature (London) 392, 160 (1998).

[3] W. Marshall, C. Simon, R. Penrose, and D. Bouwmeester, Phys. Rev. Lett. 91130401 (2003).

[4] P. F. Cohadon, A. Heidmann, and M. Pinard, Phys. Rev. Lett. 83, 3174 (1999).

[5] S. Mancini, D. Vitali, and P. Tombesi, Phys. Rev. Lett. 80, 688 (1998).

[6] V. B. Braginsky, Measurement of Weak Forces in Physics Experiments (University of Chicago, Chicago, 1977).

[7] T. J. Kippenberg, H. Rokhsari, T. Carmon, A. Scherer, and K. J. Vahala, Phys. Rev. Lett. 95, 033901 (2005).

[8] V. B. Braginsky and S. P. Vyatchanin, Phys. Lett. A 293, 228 (2002).

[9] H. G. Craighead, Science 290, 1532 (2000).

[10] X. M. H. Huang, C. A. Zorman, M. Mehregany, and M. L. Roukes, Nature (London) 421, 496 (2003).

[11] C. H. Metzger and K. Karrai, Nature (London) 432, 1002 (2004).

[12] H. Rokhsari, T. J. Kippenberg, T. Carmon, and K. J. Vahala, Opt. Express 13, 5293 (2005).

[13] V. B. Braginsky, S. E. Strigin, and S. P. Vyatchanin, Phys. Lett. A 287, 331 (2001).

[14] V.B. Braginsky and F. Khalili, Quantum Measurement (Cambridge University Press, Cambridge, England, 1992).

[15] A. Dorsel, J. D. McCullen, P. Meystre, E. Vignes, and H. Walther, Phys. Rev. Lett. 51, 1550 (1983).

[16] S. Mancini, V. Giovannetti, D. Vitali, and P. Tombesi, Phys. Rev. Lett. 88, 120401 (2002).

[17] T. Carmon, H. Rokhsari, L. Yang, T. J. Kippenberg, and K. J. Vahala, Phys. Rev. Lett. 94, 223902 (2005).

[18] K. J. Vahala, Nature (London) 424, 839 (2003).

[19] D. K. Armani, T. J. Kippenberg, S. M. Spillane, and K. J. Vahala, Nature (London) 421, 925 (2003).

[20] F. Marquardt, J. Harris, and S. Girvin, Phys. Rev. Lett. 96, 103901 (2006).

[21] V. Vuletic and S. Chu, Phys. Rev. Lett. 84, 3787 (2000).

[22] P. Maunz, T. Puppe, I. Schuster, N. Syassen, P. W. H. Pinkse, and G. Rempe, Nature (London) 428, 50 (2004).

[23] M. Zalalutdinov, A. Zehnder, A. Olkhovets, S. Turner, L. Sekaric, B. Ilic, D. Czaplewski, J. M. Parpia, and H. G. Craighead, Appl. Phys. Lett. 79, 695 (2001).

[24] H. Rokhsari and K. J. Vahala, Opt. Lett. 30, 427 (2005).

[25] O. Arcizet, P.-F. Cohadon, T. Briant, M. Pinard, and A. Heidmann, Nature (London) 444, 71 (2006).

[26] S. Gigan, H. R. Boehm, N. Paternostro, F. Blaser, G. Langer, J. B. Hertzberg, K. C. Schwab, D. Baeuerle, M. Aspelmeyer, and A. Zeilinger, Nature (London) 444, 67 (2006).

[27] D. Kleckner and D. Bouwmeester, Nature (London) 444, 75 (2006). 